\title{
Über die Schreibweise
}

Zu meiner Genugtuung kann ich feststellen, daß die Vereinheitlichung der Schreibweise medizinischer Fachwörter, für die ich mich seit Jahren eingesetzt habe, von allen mir bekannten deutschen Zeitschriften und auch von fast allen Lehrbüchern angenommen worden ist.

Für dio Schreibweise des K- und Z-Lautes in den lateinischen und griechischlateinischen Fachwörtern gelten folgende Regeln:

I. Soweit die Fachwörter in der ursprünglichen lateinischen oder griechischlateinischen Forın erscheinen, wird geschrieben:

1) c vor a, o, $u$ und vor Konsonanten:

Cataracta, Carcinoma, Anthracosis, Tuberculosis, Appendicitis acuta, Acne, Cataracta, Acrocyanosis;

$2 \mathfrak{a})$ c vor e, l, ae, oe, ue, y,

wenn es nach lateinischer Aussprache wic z gesprochen werden soll: Carcinoma, Appendicitis, Coeloma, Cystitis, Acrocyanosis;

b) $k$ vor e, i, ae (ai), oe (oi), ue, y,

wenn es nach griechischer Aussprache wie $k$ gesprochen werden soll: Keratoma, Ankylosis, Kystadenoma.

II. Soweit die Fachwörter ihrer Endung nach eingedeutscht sind, wird der Ausspracho entsprechend der K.Laut mit k, der Z-Laut mit $\mathbf{z}$ geBchritben:

Karzinom, Keratom, Koilonychie, akut, Tuberkulose; Alopezie, Karzinom, Ozon, Zyste, Zystalgie.

Diese Regel gilt auch, wenn aus dem $\mathrm{Zusammenhang} \mathrm{erkenntlich} \mathrm{ist,}$ daß Eindeutschung rorliegt:

akute Appendizitis, eiternde Akne.

Für die Betonung mancher Fachwörter lassen sich bestimmte Regeln nicht aufstellen, da sie teilweise erst in der Neuzeit von einzeinen Autoren gebildet wurden, z. B. Ochronosis von R. VIRCHOw; auch hier entscheidet der Sprachgebrauch, z. B. Ozớn, eigentlich Ŏ̌zơn = das Riechende, Duftende. 